\title{
Editorial
}

\section{Fractional Differential Equations}

\author{
Fawang Liu, ${ }^{1}$ Mark M. Meerschaert, ${ }^{2}$ Shaher Momani, ${ }^{3}$ \\ Nikolai N. Leonenko, ${ }^{4}$ Wen Chen, ${ }^{5}$ and Om P. Agrawal ${ }^{6}$ \\ ${ }^{1}$ School of Mathematical Sciences, Queensland University of Technology, P.O. Box 2434, \\ Brisbane, Qeensland 4001, Australia \\ ${ }^{2}$ Department of Statistics and Probability, Michigan State University, A416 Wells Hall, \\ East Lansing, MI 48823, USA \\ ${ }^{3}$ Department of Mathematics, Mu'tah University, P.O. Box 7, Mu'tah 61710, Jordan \\ ${ }^{4}$ School of Mathematics, Cardiff University, Cardiff CF2 4YH, UK \\ ${ }^{5}$ Department of Engineering Mechanics, Hohai University, Xikang Road Number 1, \\ Nanjing, Jiangsu 210098, China \\ ${ }^{6}$ Department of Mechanical Engineering and Energy Processes, Southern Illinois University, \\ Carbondale, IL 62901, USA
}

Correspondence should be addressed to Fawang Liu, f.liu@qut.edu.au

Received 13 April 2010; Accepted 13 April 2010

Copyright (C) 2010 Fawang Liu et al. This is an open access article distributed under the Creative Commons Attribution License, which permits unrestricted use, distribution, and reproduction in any medium, provided the original work is properly cited.

It is my pleasure to present this special issue of the International Journal of Differential Equations dedicated to Fractional Differential Equations (FDEs).

Fractional differential equations are generalizations of ordinary differential equations to an arbitrary (noninteger) order. Fractional differential equations have attracted considerable interest because of their ability to model complex phenomena. These equations capture nonlocal relations in space and time with power-law memory kernels. Due to the extensive applications of FDEs in engineering and science, research in this area has grown significantly all around the world.

This special issue of Fractional Differential Equations consists of one review article (paper 1) and 12 original articles covering various aspects of FDEs and their applications by the prominent researchers in the field.

The review article surveys the properties of a transcendental function of the Wright type, nowadays known as the M-Wright function, entering as a probability density in a relevant class of self-similar stochastic processes. The second paper presents a computationally effective numerical method for the time-space fractional Fokker-Planck equations with a nonlinear source term, together with a stability and convergence analysis of the numerical method. 
The third paper uses He's variational iteration method for solving the fractional Riccati differential equation. The fourth paper proposes a fractional iteration method and has been applied to study the Hirota-Satsuma coupled KdV of time-fractional-order equation.

The fifth paper develops a formulation for fractional time-optimal control problems. The sixth paper derives conditions for the speed of spread of solutions of fractional scalar reaction-diffusion equations to be finite or infinite. The seventh paper studies the Riesz fractional derivatives for the Korteweg-de Vries solitons and proves that these Riesz potentials and their Hilbert transforms are linearly independent solutions of a SturmLiouville problem.

The eighth paper gives the analytical solution of the linear fractionally damped oscillator equation by means of Laplace transform. The ninth paper proves the existence and uniqueness of a positive solution to the nonzero boundary value problem for a coupled system of fractional differential equations. The tenth paper gives sufficient conditions on the existence of a periodic solution for a class of compound singular fractional differential systems with delay, involving Nishimoto fractional derivative. The eleventh paper discusses the existence and uniqueness of the solutions of the nonhomogeneous linear differential equations of arbitrary positive real order by using the fractional B-Splines wavelets and the Mittag-Leffler function. The twelfth paper proves the existence of solutions of the fractional Langevin equation with two different fractional orders in a Banach space using the contraction mapping principle and Krasnoselskii's fixed point theorem. The final paper addresses the selection and meaning of variable-order operators for dynamic modelling.

Thus, this special issue provides a wide spectrum of current research in the area of FDEs, and I hope that experts in this and related fields would find it useful.

Finally, I would like to acknowledge the guest editors Mark M. Meerschaert, Shaher Momani, Nikolai N. Leonenko, We Chen, and Om P. Agrawal for their assistance in producing this special edition within the specified timeframe.

Fawang Liu

Mark M. Meerschaert

Shaher Momani

Nikolai N. Leonenko

Wen Chen

Om P. Agrawal 


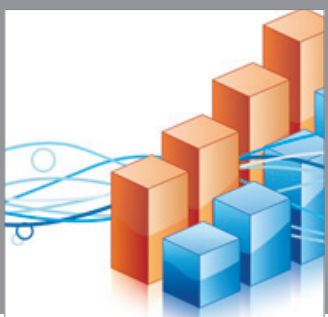

Advances in

Operations Research

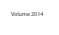

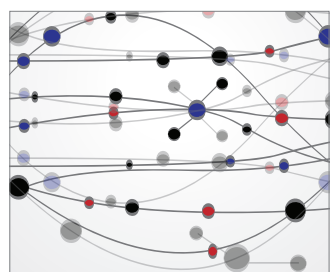

\section{The Scientific} World Journal
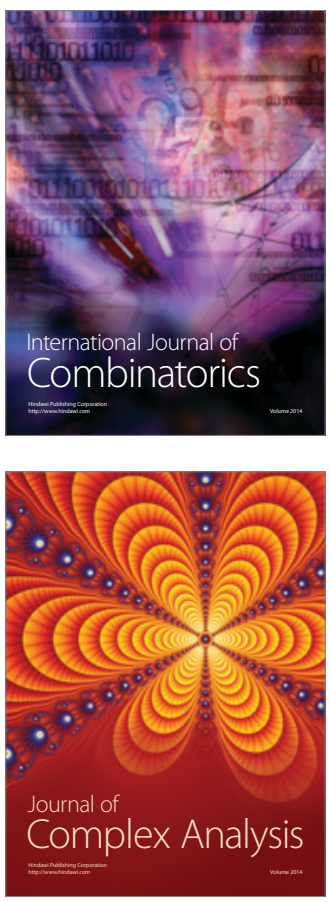

International Journal of

Mathematics and

Mathematical

Sciences
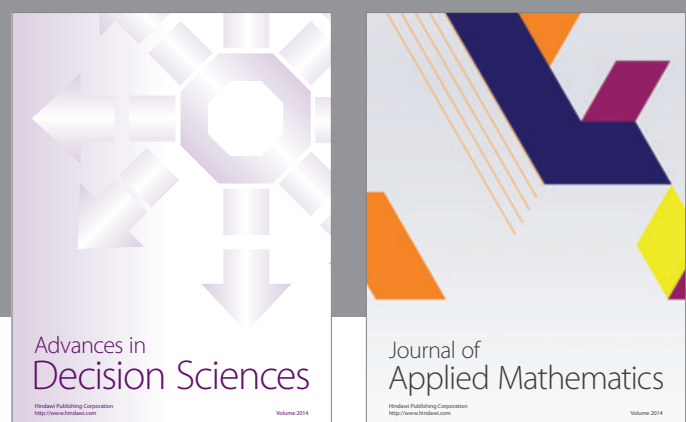

Journal of

Applied Mathematics
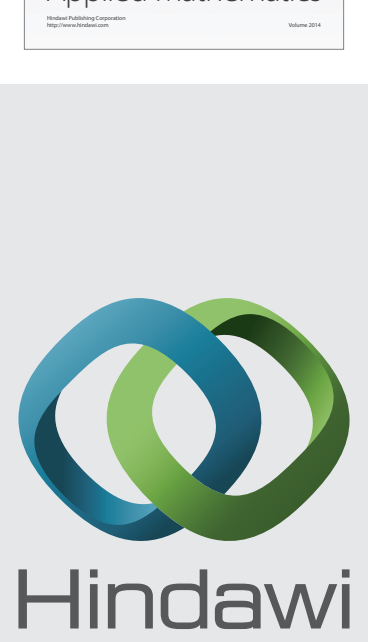

Submit your manuscripts at http://www.hindawi.com
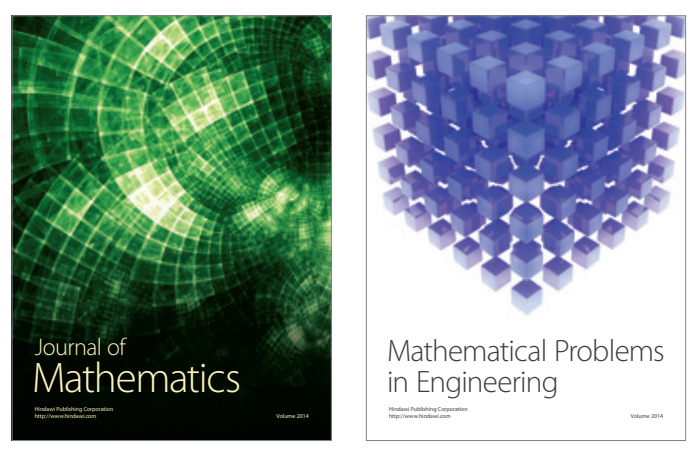

Mathematical Problems in Engineering
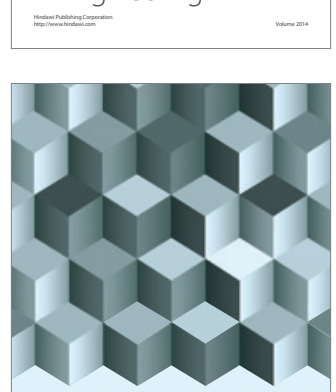

Journal of

Function Spaces
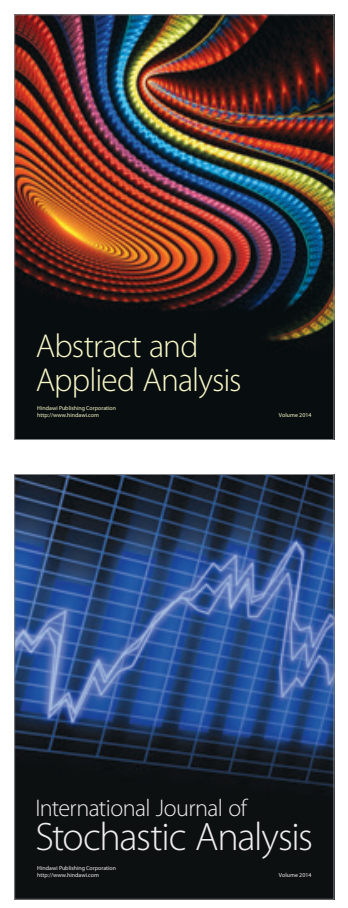

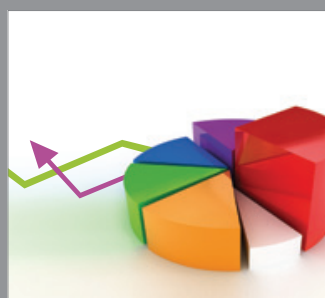

ournal of

Probability and Statistics

Promensencen
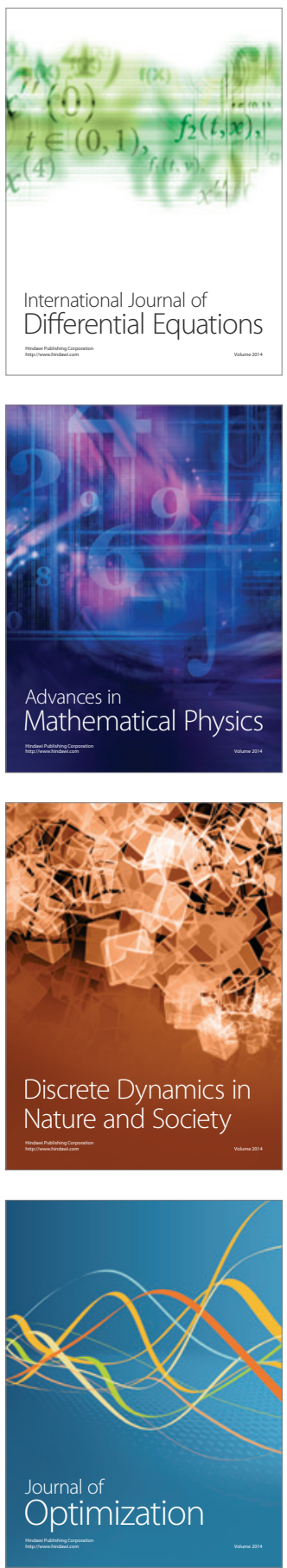